\title{
Stability and structure of quark matter in a molecular dynamics framework
}

\author{
Yuka Akimura $^{a b}$, Toshiki Maruyama ${ }^{b}$, Naotaka Yoshinaga ${ }^{a}$, and Satoshi Chiba ${ }^{b}$ \\ ${ }^{a}$ Department of physics, Saitama University, Sakura-Ku, Saitama-Shi, 338-8570, Japan \\ bAdvanced Science Research Center, Japan Atomic Energy Research Institute, Tokai, \\ Ibaraki 319-1195, Japan
}

\begin{abstract}
We study stability and structure of quark matters as a function of density in a framework of molecular dynamics (MD). Using appropriate effective interactions and the frictional cooling method, we search for the minimum energy of the system. Transition from confined to deconfined phase is observed at densities of $2-3 \rho_{0}$, where $\rho_{0}$ is the nuclear matter saturation density. The $u d s$ matter becomes more stable than the charge-neutral $u d$ matter at $3 \rho_{0}$, but the $u d d$ matter is the most stable even at high density.
\end{abstract}

\section{Introduction}

More than twenty years ago Bodmer and later Witten put a hypothesis that the absolute ground state of strongly interacting matter is the deconfined state of quark matter consisting of an equal proportion of up, down, and strange quarks called strange quark matter even at zero temperature and zero pressure 12 .

Recently some exotic baryon systems including strange quarks were discovered. For example, the penta-quark, called $\Theta^{+}$, consisting of 5 quarks ( uudd $\bar{s}$ ) with an extraordinary small width $\sim 25 \mathrm{MeV}$, was discovered at SPring8 [3]. At KEK, a strongly bounded kaonic system, ppn $K^{-}$[45], which was predicted from an AMD calculation [6] to form a high density system of $10 \rho_{0}$, was discovered. From an astrophysical point of view, there is a possibility that the strange quark matter is realized at the center of neutron stars and/or quark stars [7]. At high baryon density such as in the core of these stars, quark gluon plasma (QGP) may exist and confined baryon and deconfined baryon states may coexist [89]. Searching for the energy region in which the hadron to quark transition occurs and the strange quark matter becomes stable is one of the most interesting topics in high energy hadron physics.

In this paper we study the stability of quark matter at finite baryon density at zero temperature using a molecular dynamics method (MD). In this framework it is not necessary to assume whether the state of quarks is confined or deconfined and their mixed state can also be treated [10]. 

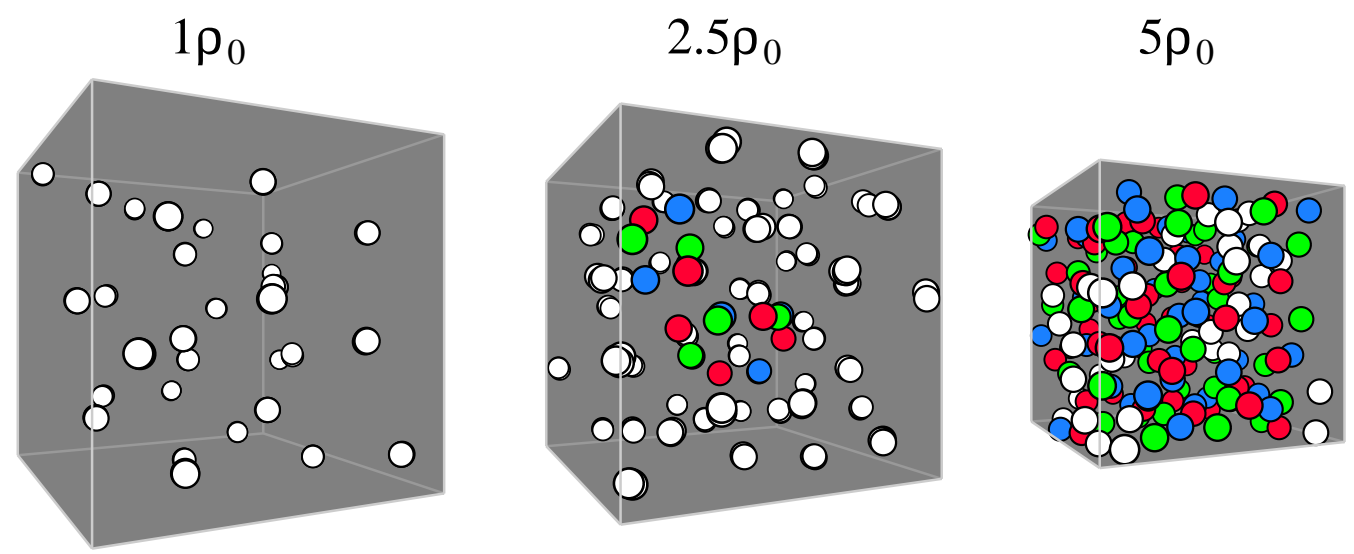

Figure 1. Snapshots for the udd matter after cooling. The white circles denote confined quarks and colored circles are deconfined quarks.

\section{Molecular dynamics for quark system}

We define the total wave function of a system $\Psi$ with baryon number $A$ as a direct product of single particle wave functions with a Gaussian wave packet in coordinate space and a state vector $\chi_{i}$ with a fixed spin orientation, flavor and color,

$\Psi=\prod_{i=1}^{3 A} \frac{1}{\left(\pi L^{2}\right)^{3 / 4}} \exp \left[-\frac{\left(\mathbf{r}-\mathbf{R}_{i}\right)^{2}}{2 L^{2}}+\frac{i}{\hbar} \mathbf{P}_{i} \mathbf{r}\right] \chi_{i}$.

The antisymmetrization of the wave function is neglected, but treated phenomenologically by introducing a Pauli potential which acts as a repulsive force between quarks of the same color, flavor and spin orientation. The Hamiltonian is expressed as $H=H_{0}+V_{\text {Pauli }}-T_{\text {spur }}$, where $T_{\text {spur }}$ is a spurious zero point kinetic energy of clusters as described in [11]. The original Hamiltonian $H_{0}$ and $V_{\text {Pauli }}$ are explicitly expressed as,

$$
\begin{aligned}
H_{0} & =\left\langle\Psi\left|\sum_{i}\left(\frac{\hat{\mathbf{p}}_{i}^{2}}{2 m_{i}}+m_{i}\right)+\frac{1}{2} \sum_{j \neq i}\left(-\sum_{a=1}^{8} \frac{\lambda_{i}^{a} \lambda_{j}^{a}}{4}\left(K \hat{r}-\frac{\alpha_{s}}{\hat{r}}\right)+\hat{V}_{\text {meson }}\right)\right| \Psi\right\rangle, \\
\hat{V}_{\text {meson }} & \equiv-\frac{g_{\sigma q}^{2}}{4 \pi} \frac{e^{-\mu_{\sigma} \hat{r}}}{\hat{r}}+\frac{g_{\omega q}^{2}}{4 \pi} \frac{e^{-\mu_{\omega} \hat{r}}}{\hat{r}}, \\
V_{\text {Pauli }} & \equiv \frac{1}{2} \sum_{j \neq i} C_{p}\left(\frac{\hbar}{q_{0} p_{0}}\right)^{3} \exp \left[-\frac{\left(\mathbf{R}_{i}-\mathbf{R}_{j}\right)^{2}}{2 q_{0}^{2}}-\frac{\left(\mathbf{P}_{i}-\mathbf{P}_{j}\right)^{2}}{2 p_{0}^{2}}\right],
\end{aligned}
$$

where $\hat{r}=\left|\hat{\mathbf{r}}_{i}-\hat{\mathbf{r}}_{j}\right|$ and $\lambda_{i}^{a}=\left\langle\chi_{i}\left|\lambda^{a}\right| \chi_{i}\right\rangle$. We use $\alpha_{s}=1.25$ (the QCD fine structure constant), $K=900 \mathrm{MeV} / \mathrm{fm}$ (string tension), constituent quark masses $m_{u, d}=300 \mathrm{MeV}$ and $m_{s}=500 \mathrm{MeV}$ throughout this simulation which are typical values of parameters in quark models. The product of Gell-Mann matrices for different colors and for same colors are $\left\langle\sum_{a=1}^{8} \lambda_{i}^{a} \lambda_{j}^{a}\right\rangle=-\frac{2}{3}$ and $\frac{4}{3}$, respectively. However, since the antisymmetrization is neglected in our model, we multiply those values by 4 to effectively include the exchange term of color interaction. To introduce the nuclear force between white (colorless) baryons, we 


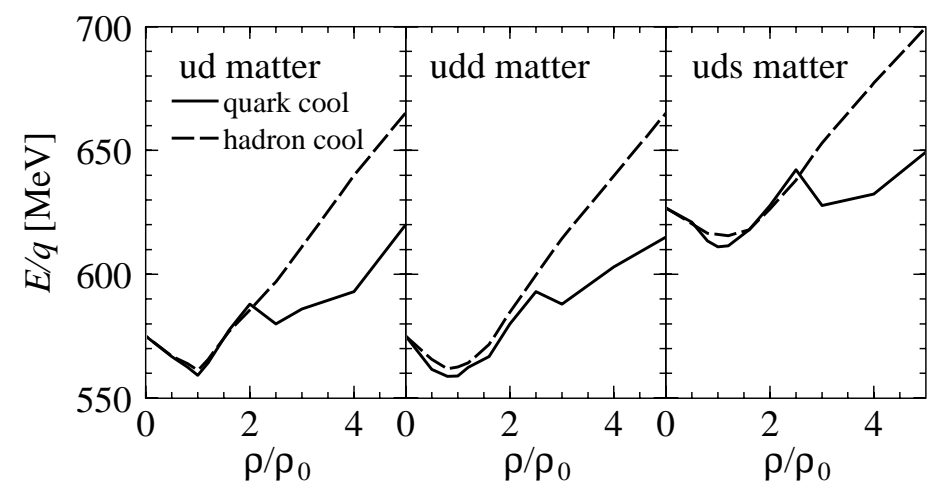

Figure 2. Energy per quark for three kinds of matter. Dashed lines denote the result of "hadron-cooling" in which 3 quarks always form a colorless hadron, while solid lines are those of ordinary "quarkcooling" without any constraint.

employ the meson exchange potential $\hat{V}_{\text {meson }}$ acting between quarks. The $\sigma$ and $\omega$ mesonquark coupling constants are $g_{\sigma q}=3.42$ and $g_{\omega q}=9.0$, the meson masses are $\mu_{\sigma}=300 \mathrm{MeV}$ and $\mu_{\omega}=782 \mathrm{MeV}$, respectively. The parameters in the Pauli potential $V_{\text {Pauli }}$ are determined to reproduce the kinetic energy of the Fermi gas [12. In the present study we adopt $C_{p}=131 \mathrm{MeV}, q_{0}=1.8 \mathrm{fm}, p_{0}=120 \mathrm{MeV}$ for $u$ and $d$ quarks and $C_{p}=79 \mathrm{MeV}$ for $s$ quarks. The size of the quark wave packet, $L$, is taken to be $0.43 \mathrm{fm}$, whereas $L^{\mathrm{eff}}=0.7 \mathrm{fm}$ is used in $\left\langle\hat{V}_{\text {meson }}\right\rangle$ to take into account of the baryon size [10].

\section{Quark matter properties}

Here we investigate the ground state (zero temperature) of matter consisting of quarks. As an initial condition we distribute "hadrons" (made from 3 quarks with different colors) randomly in a cell with a periodic boundary condition. In the case of baryon density, e.g., of $1 \rho_{0}, 90$ quarks are put in a cell of a size $5.6 \mathrm{fm}$. To search for the minimum energy configuration of the system, we solve the cooling equations of motion with friction terms as in [12. The simulations are performed for " $u d$ matter" (with same numbers of $u$ and $d$ quarks), "udd matter" (number of $d$ quarks is twice that of $u$ quarks) and " $u d s$ matter" (with same numbers of $u, d$, and $s$ quarks).

Snapshots of the $u d d$ matter for different baryon densities are displayed in Fig. 1. All quarks are confined as hadrons at baryon density of $1 \rho_{0}$. At $2.5 \rho_{0}$, a partly deconfined state can be seen, whereas almost all quarks are deconfined at $5 \rho_{0}$.

The ground state energies of matter are shown with solid lines in Fig. 2. The dashed lines show results with a constraint that $\mathbf{R}_{i}$ of 3 quarks in each initial "hadron" should always be equal (to keep hadrons by hand). Here we call this calculation "hadron-cooling", while the normal one we call "quark-cooling". At lower density, the energy of the system by hadron-cooling agrees that by quark-cooling. This means that quarks are confined in hadrons as seen in Fig. 1. Note that the saturation of hadronic matter is realized around $1 \rho_{0}$ for the first time in this kind of quark MD simulation. The energies of the ud matter and the $u d d$ matter are almost the same. This is because the Pauli potential is not fully effective for "hadrons" and $\rho$ meson exchange is missing. At densities of $2-3 \rho_{0}$, transition to deconfined phase occurs. In our simulation, the decrease of the kinetic energy and the increase of the potential energy act oppositely during the deconfinement. The softening of the latter effect may be the origin of the transition at higher densities.

Figure 3 shows comparison of energy among the $u d$ matter with relativistic electrons, 


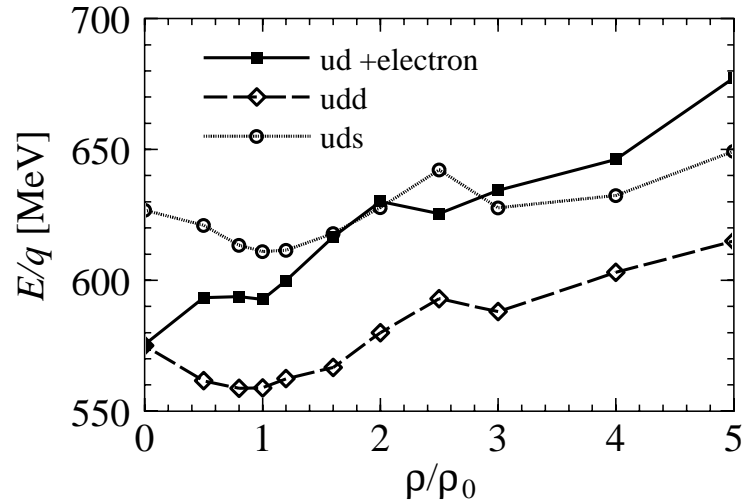

Figure 3. Baryon density dependence of the energy per quark for three kinds of matter under charge-neutral condition.

the udd matter and the uds matter under charge neutral condition. Although the $u d s$ matter is the most unstable at low density, it becomes more stable than the $u d$ matter as deconfinement occurs at $3 \rho_{0}$. The $u d d$ matter, however, is the most stable over all ranges in our present result.

\section{Summary}

Quark many-body systems were studied in a framework of MD. It has been found that hadron matter undergoes a phase transition to the quark matter as baryon density increases. At low density, the saturation for the $u d$ matter is realized by introducing $\hat{V}_{\text {meson. }}$. The $u d d$ and $u d s$ matter, however, also show the similar saturation because exactly the same meson exchange potentials are used among all quarks. To improve this, a flavor-dependent potential like $\rho$ meson exchange potential is needed. The $u d s$ matter becomes more stable than the charge-neutral $u d$ matter at $3 \rho_{0}$. But the $u d d$ matter is the most stable even at high density. We expect more reasonable results if $\rho$ meson exchange potential is included in the future.

\section{REFERENCES}

1. A. R. Bodmer, Phys. Rev. D4 (1971) 1601.

2. E. Witten, Phys. Rev. D30 (1984) 272.

3. T. Nakano et al., Phys. Rev. Lett. 91 (2003) 012002.

4. M. Iwasaki et al., nucl-ex/0310018,

5. T. Suzuki et al., Phys. Rev. Lett. B597 (2004) 263.

6. A. Doté, H. Horiuchi, Y. Akaishi and T. Yamazaki. nucl-th/0309062.

7. J. J. Drake et al., Astrophys. J. 572 (2002) 996.

8. I. N. Mishustin, L. M. Satarov, H. Stöcker and W. Greiner, Phys. Rev. C62 (2000) 034901.

9. S. K. Ghosh and S. C. Phatak, Phys. Rev. C58 (1998) 1714.

10. T. Maruyama and T. Hatsuda, Phys. Rev. C61 (2000) 062201.

11. A. Ono, H. Horiuchi, T. Maruyama and A. Ohnishi, Prog. Theor. Phys. 87 (1992) 1185.

12. T. Maruyama, K. Niita, K. Oyamatsu, T. Maruyama, S. Chiba and A. Iwamoto, Phys. Rev. C57 (1997) 655. 